\title{
Answer Keys to Chapter Exercises
}

EXERCISE 5.1 QUESTION FRAMEWORK AND BEST STUDY DESIGN FOR A SYSTEMATIC REVIEW QUESTION

\begin{tabular}{|c|c|c|c|}
\hline $\begin{array}{l}\text { 1a. Foreground Research } \\
\text { Question }\end{array}$ & Framework & Question Type & $\begin{array}{l}\text { Best Study Design(s) for } \\
\text { Answering Question }\end{array}$ \\
\hline \multirow{5}{*}{$\begin{array}{l}\text { What is the economic } \\
\text { evidence on self- } \\
\text { measured blood } \\
\text { pressure (SMBP) } \\
\text { monitoring interven- } \\
\text { tions for high blood } \\
\text { pressure control (Jacob } \\
\text { et al., 2017)? }\end{array}$} & $\begin{array}{l}\mathbf{P}=\text { patients with high blood } \\
\text { pressure }\end{array}$ & \multirow[t]{4}{*}{ Economic Evaluation } & \multirow{5}{*}{$\begin{array}{l}\text { Randomized controlled } \\
\text { trials with methodolo- } \\
\text { gies including } \\
\text { cost-effectiveness and } \\
\text { or cost-benefit }\end{array}$} \\
\hline & I = SMBP monitoring & & \\
\hline & $\mathbf{C}=$ comparison $(\mathrm{s})$ & & \\
\hline & $\begin{array}{l}\mathbf{0} \text { = cost-effectiveness of } \\
\text { SMBP alone and/or with } \\
\text { additional care measures: } \\
\text { change in emergency room } \\
\text { visits, medication, inpatient } \\
\text { and outpatient services } \\
\text { costs }\end{array}$ & & \\
\hline & $\begin{array}{l}\mathbf{C}=\text { high blood pressure in the } \\
\text { United States }\end{array}$ & & \\
\hline $\begin{array}{l}\text { 1b. Foreground Research } \\
\text { Question }\end{array}$ & Framework & Question Type & $\begin{array}{l}\text { Best Study Design(s) for } \\
\text { Answering Question }\end{array}$ \\
\hline \multirow{4}{*}{$\begin{array}{l}\text { What is the effect of } \\
\text { breathing exercises on } \\
\text { chronic asthma } \\
\text { management by adults } \\
\text { (Santino et al, 2020)? }\end{array}$} & $\begin{array}{l}\mathbf{P}=\text { adults with chronic } \\
\text { asthma }\end{array}$ & \multirow[t]{4}{*}{$\begin{array}{l}\text { Effectiveness of } \\
\text { Intervention }\end{array}$} & \multirow{4}{*}{$\begin{array}{l}\text { Randomized controlled } \\
\text { trials, non-randomized } \\
\text { trials }\end{array}$} \\
\hline & I = breathing exercises & & \\
\hline & $\begin{array}{l}\mathbf{C}=\text { no breathing exercises or } \\
\text { asthma education }\end{array}$ & & \\
\hline & $\begin{array}{l}\mathbf{0}=\text { asthma control/symptom } \\
\text { management, exacerbation, } \\
\text { hospitalization, quality of } \\
\text { life }\end{array}$ & & \\
\hline $\begin{array}{l}\text { 1c. Foreground Research } \\
\text { Question }\end{array}$ & Framework & Question Type & $\begin{array}{l}\text { Best Study Design(s) for } \\
\text { Answering Question }\end{array}$ \\
\hline \multirow{3}{*}{$\begin{array}{l}\text { What are the impacts of } \\
\text { acculturation, } \\
\text { Marianismo, and } \\
\text { religiosity on perinatal } \\
\text { depression in Latina } \\
\text { women (Lara-Cinisomo } \\
\text { et al., 2019)? }\end{array}$} & $\mathbf{P}=$ Latina women & \multirow[t]{3}{*}{ Etiology, Risk Factor(s) } & \multirow{3}{*}{$\begin{array}{c}\text { Cohort, cross-sectional, } \\
\text { case-control studies }\end{array}$} \\
\hline & $\begin{array}{l}\mathbf{E}=\text { acculturation, Marianismo, } \\
\text { and religiosity }\end{array}$ & & \\
\hline & $\mathbf{0}=$ perinatal depression & & \\
\hline
\end{tabular}


EXERCISE 5.I QUESTION FRAMEWORK AND BEST STUDY DESIGN FOR A SYSTEMATIC REVIEW QUESTION (continued)

\begin{tabular}{|c|c|c|c|}
\hline $\begin{array}{l}\text { 1d. Foreground Research } \\
\text { Question }\end{array}$ & Framework & Question Type & $\begin{array}{l}\text { Best Study Design(s) for } \\
\text { Answering Question }\end{array}$ \\
\hline \multirow{3}{*}{$\begin{array}{l}\text { What is the experience of } \\
\text { using videoconferenc- } \\
\text { ing by registered } \\
\text { nurses and/or midwives } \\
\text { for practice (Penny } \\
\text { et al., 2018? }\end{array}$} & $\begin{array}{l}\mathbf{P}=\text { registered nurses and } \\
\text { midwives }\end{array}$ & \multirow[t]{3}{*}{ Qualitative Experience } & \multirow[t]{3}{*}{ Qualitative studies } \\
\hline & $\mathbf{I}=$ videoconferencing & & \\
\hline & $\mathbf{C}=$ nursing practice & & \\
\hline $\begin{array}{l}\text { 1e. Foreground Research } \\
\text { Question }\end{array}$ & Framework & Question Type & $\begin{array}{l}\text { Best Study Design(s) for } \\
\text { Answering Question }\end{array}$ \\
\hline \multirow{4}{*}{$\begin{array}{l}\text { In rural communities, } \\
\text { what is the perspective } \\
\text { of family caregivers of } \\
\text { transitional palliative } \\
\text { care patients on } \\
\text { deprescribing } \\
\text { compared to that of } \\
\text { family caregivers of } \\
\text { patients on palliative } \\
\text { care units? }\end{array}$} & $\mathbf{S}=$ Rural communities & \multirow[t]{4}{*}{ Qualitative Experience } & \multirow{5}{*}{$\begin{array}{c}\text { Qualitative or Mixed } \\
\text { Methods studies }\end{array}$} \\
\hline & $\begin{array}{l}\mathbf{P}=\text { Transitional palliative care } \\
\text { family caregivers }\end{array}$ & & \\
\hline & $\begin{array}{l}\text { I = Deprescribing via } \\
\text { transitional palliative care }\end{array}$ & & \\
\hline & $\begin{array}{r}\mathbf{C}=\text { Deprescribing via } \\
\text { palliative care unit }\end{array}$ & & \\
\hline & $\begin{array}{l}\mathbf{E}=\text { Family caregivers' } \\
\text { perceptions and experiences }\end{array}$ & & \\
\hline
\end{tabular}

$\mathrm{PICOC}$ is $\mathrm{P}=$ patient/population, problem of interest, $\mathrm{I}=$ intervention, $\mathrm{C}=\operatorname{comparison}(\mathrm{s}), \mathrm{O}=$ outcome, $\mathrm{C}=$ context; $\mathrm{PICO}$ is $\mathrm{P}=$ patient/population, problem of interest, $\mathrm{I}=$ intervention, $\mathrm{C}=\operatorname{comparison}(\mathrm{s}), \mathrm{O}=$ outcome;

$\mathrm{PEO}$ is $\mathrm{P}=$ patient/population, $\mathrm{E}=$ exposure of risk, $\mathrm{O}=$ outcome;

$\mathrm{PIC}$ is $\mathrm{P}=$ patient/population, $\mathrm{I}=$ phenomena of interest, $\mathrm{C}=$ context;

SPICE is $\mathrm{S}=$ setting, $\mathrm{P}=$ perspective, $\mathrm{I}=$ phenomena of interest, $\mathrm{C}=$ comparison, $\mathrm{E}=$ evaluation.

\section{REFERENCES}

Jacob, V., Chattopadhyay, S. K., Proia, K. K., Hopkins, D. P., Reynolds, J., Thota, A. B., Jones, C. D., Lackland, D. T., Rask, K. J., Pronk, N. P., Clymer, J. M., Goetzel, R. Z., \& Community Preventive Services Task Force. (2017). Economics of self-measured blood pressure monitoring: a community guide systematic review. American Journal of Preventive Medicine, 53(3), e105-e113. https://doi.org/10.1016/j.amepre.2017.03.002. Epub 2017 Aug 14. PMID: 28818277; PMCID: PMC5657494.

Lara-Cinisomo, S., Wood, J., \& Fujimoto, E. M. (2019). A systematic review of cultural orientation and perinatal depression in Latina women: are acculturation, Marianismo, and religiosity risks or protective factors? Archives of Women's Mental Health, 22(5), 557-567. https://doi.org/10.1007/s00737-018-0920-4

Penny, R. A., Bradford, N. K., \& Langbecker, D. (2018). Registered nurse and midwife experiences of using videoconferencing in practice: A systematic review of qualitative studies. Journal of Clinical Nursing, 27(5-6), e739-e752. https://doi.org/10.1111/jocn.14175

Santino, T. A., Chaves, G. S., Freitas, D. A., Fregonezi, G. A., \& Mendonça, K. M. (2020). Breathing exercises for adults with asthma. Cochrane Database of Systemic Reviews, 3(3), Cd001277. https://doi.org/10.1002/14651858. CD001277.pub4 
EXERCISE 7.1 CONTROLLED VOCABULARY (E.G., SUBJECT HEADINGS) FOR TEXT WORDS

\begin{tabular}{|c|c|c|c|c|}
\hline Text Words & $\begin{array}{c}\text { MeSH Database } \\
\text { https://www. } \\
\text { ncbi.nlm.nih. } \\
\text { gov/mesh }\end{array}$ & $\begin{array}{c}\text { MeSH in } \\
\text { MEDLINE (Ovid) }\end{array}$ & $\begin{array}{c}\text { Subject } \\
\text { Headings in } \\
\text { CINAHL (EBSCO) }\end{array}$ & $\begin{array}{c}\text { Comment on What Was } \\
\text { Discovered }\end{array}$ \\
\hline AIDS & $\begin{array}{l}\text { Acquired } \\
\text { Immunodeficiency } \\
\text { Syndrome }\end{array}$ & $\begin{array}{l}\text { Acquired } \\
\text { Immunodefi- } \\
\text { ciency } \\
\text { Syndrome }\end{array}$ & $\begin{array}{l}\text { Acquired } \\
\text { Immunodefi- } \\
\text { ciency Syndrome }\end{array}$ & \\
\hline $\begin{array}{l}\text { Alternative } \\
\text { Therapy }\end{array}$ & $\begin{array}{l}\text { Complementary } \\
\text { Therapies }\end{array}$ & $\begin{array}{l}\text { Complementary } \\
\text { Therapies }\end{array}$ & $\begin{array}{l}\text { Alternative } \\
\text { Therapies }\end{array}$ & \\
\hline $\begin{array}{l}\text { Anti-Inflammatory } \\
\text { Agents }\end{array}$ & $\begin{array}{l}\text { Anti-Inflammatory } \\
\text { Agents }\end{array}$ & $\begin{array}{l}\text { Anti-Inflammatory } \\
\text { Agents }\end{array}$ & $\begin{array}{l}\text { Anti-Inflammatory } \\
\text { Agents }\end{array}$ & \\
\hline Bed Rest & Bed Rest & Bed Rest & Bed Rest & \\
\hline $\begin{array}{l}\text { Cardiac Catheter- } \\
\text { ization }\end{array}$ & $\begin{array}{l}\text { Cardiac } \\
\quad \text { Catheterization }\end{array}$ & $\begin{array}{l}\text { Cardiac Catheter- } \\
\text { ization }\end{array}$ & $\begin{array}{l}\text { Heart } \\
\text { Catheterization }\end{array}$ & $\begin{array}{l}\text { Realize that MeSH terms are } \\
\text { used in the MEDLINE } \\
\text { database regardless of } \\
\text { whether an investigator is } \\
\text { searching MEDLINE via the } \\
\text { Ovid search retrieval system } \\
\text { or within PubMed. }\end{array}$ \\
\hline Coronavirus 2019 & $\begin{array}{l}\text { Severe Acute } \\
\text { Respiratory } \\
\text { Syndrome } \\
\text { Coronavirus 2 }\end{array}$ & $\begin{array}{l}\text { Coronavirus } \\
\text { Infections }\end{array}$ & $\begin{array}{l}\text { Coronavirus disease } \\
2019 \\
\text { Use: COVID-19 }\end{array}$ & $\begin{array}{l}\text { The MeSH Database yielded } \\
\text { many MeSH terms on the } \\
\text { search: Coronavirus } 2019 \\
\text { and the most relevant for } \\
\text { my search was the term: } \\
\text { severe acute respiratory } \\
\text { syndrome Coronavirus 2. } \\
\text { Surprisingly, in MEDLINE } \\
\text { (Ovid) } 1946 \text { to October } \\
\text { Week } 42020 \text {, the most } \\
\text { relevant term was Coronavi- } \\
\text { rus Infections. Even typing } \\
\text { in the term Severe Acute } \\
\text { Respiratory Syndrome } \\
\text { Coronavirus } 2 \text { in MEDLINE } \\
\text { (Ovid) did not reveal the } \\
\text { term found in PubMed: } \\
\text { Severe Acute Respiratory } \\
\text { Syndrome Coronavirus } 2\end{array}$ \\
\hline Cot Death & Sudden Infant Death & $\begin{array}{l}\text { Sudden Infant } \\
\text { Death }\end{array}$ & $\begin{array}{l}\text { Sudden Infant } \\
\text { Death }\end{array}$ & \\
\hline CPOE & $\begin{array}{l}\text { Medical Order Entry } \\
\text { Systems }\end{array}$ & $\begin{array}{l}\text { Medical Order } \\
\text { Entry Systems }\end{array}$ & $\begin{array}{l}\text { Electronic Order } \\
\text { Entry }\end{array}$ & \\
\hline $\begin{array}{l}\text { Hospital } \\
\quad \text { Readmission }\end{array}$ & Patient Readmission & $\begin{array}{l}\text { Patient Readmis- } \\
\text { sion }\end{array}$ & Readmission & \\
\hline
\end{tabular}


EXERCISE 7.1 CONTROLLED VOCABULARY (E.G., SUBJECT HEADINGS) FOR TEXT WORDS (continued)

\begin{tabular}{|c|c|c|c|c|}
\hline Text Words & $\begin{array}{c}\text { MeSH Database } \\
\text { https://www. } \\
\text { ncbi.nlm.nih. } \\
\text { gov/mesh }\end{array}$ & $\begin{array}{c}\text { MeSH in } \\
\text { MEDLINE (Ovid) }\end{array}$ & $\begin{array}{c}\text { Subject } \\
\text { Headings in } \\
\text { CINAHL (EBSCO) }\end{array}$ & $\begin{array}{c}\text { Comment on What Was } \\
\text { Discovered }\end{array}$ \\
\hline Kangaroo Care & $\begin{array}{l}\text { Kangaroo-Mother } \\
\text { Care Method }\end{array}$ & $\begin{array}{l}\text { Kangaroo-Mother } \\
\text { Care Method }\end{array}$ & Kangaroo Care & $\begin{array}{l}\text { In MEDLINE through Ovid, } \\
\text { several other subject terms } \\
\text { appear that may be useful for } \\
\text { a search on Kangaroo Care. } \\
\text { Infant care } \\
\text { Mother-child relations }\end{array}$ \\
\hline $\begin{array}{l}\text { Metabolic } \\
\text { Syndrome }\end{array}$ & Metabolic Syndrome & $\begin{array}{l}\text { Metabolic } \\
\text { Syndrome }\end{array}$ & $\begin{array}{l}\text { Metabolic } \\
\quad \text { Syndrome X }\end{array}$ & \\
\hline Nurse Burnout & $\begin{array}{l}\text { Burnout, Profes- } \\
\text { sional }\end{array}$ & $\begin{array}{l}\text { Burnout, } \\
\text { Professional }\end{array}$ & $\begin{array}{l}\text { Burnout, profes- } \\
\text { sional }\end{array}$ & $\begin{array}{l}\text { In MEDLINE through Ovid, a } \\
\text { couple of other subject } \\
\text { terms appear that may be } \\
\text { useful for a search on Nurse } \\
\text { Burnout: } \\
\text { Job satisfaction } \\
\text { Workplace }\end{array}$ \\
\hline Nursing Students & Students, Nursing & Students, Nursing & Students, Nursing & $\begin{array}{l}\text { In CINAHL, the thesaurus } \\
\text { term for nursing students } \\
\text { has a plus sign (+) in front } \\
\text { of it denoting there are } \\
\text { articles for subheadings of } \\
\text { Nursing Students, such as } \\
\text { the following: } \\
\text { Students, Nurse Midwifery } \\
\text { Students, Nursing, } \\
\text { Baccalaureate } \\
\text { Students, Nursing, Male }\end{array}$ \\
\hline $\begin{array}{l}\text { Qualitative } \\
\text { Research }\end{array}$ & Qualitative Research & $\begin{array}{l}\text { Qualitative } \\
\text { Research }\end{array}$ & Qualitative Studies & \\
\hline Tysabri & Natalizumab & Natalizumab & Natalizumab & $\begin{array}{l}\text { Searches in the MeSH } \\
\text { Database, MEDLINE (Ovid), } \\
\text { and CINAHL (EBSCO) for } \\
\text { the brand name drug, Tysabri } \\
\text { all point toward using the } \\
\text { generic drug name: } \\
\text { natalizumab for searches }\end{array}$ \\
\hline $\begin{array}{r}\text { Vitamin B12 } \\
\text { Deficiency }\end{array}$ & $\begin{array}{r}\text { Vitamin B12 } \\
\text { Deficiency }\end{array}$ & $\begin{array}{r}\text { Vitamin B12 } \\
\text { Deficiency }\end{array}$ & $\begin{array}{r}\text { Vitamin B12 } \\
\text { Deficiency }\end{array}$ & $\begin{array}{l}\text { It may be wise to use the } \\
\text { Subheading Pernicious } \\
\text { anemia as there are two } \\
\text { categories of articles under } \\
{\text { Vitamin } B_{12} \text { Deficiency in }} \\
\text { the MeSH Database and } \\
\text { MEDLINE (Ovid). } \\
\text { Anemia, pernicious } \\
\text { Subacute combined } \\
\text { degeneration }\end{array}$ \\
\hline
\end{tabular}

CINAHL, Cumulative Index to Nursing and Allied Health Literature; CPOE, computerized provider order entry; MEDLINE, Medical Literature Analysis and Retrieval System Online; MeSH, Medical Subject Headings. 
EXERCISE 7.2 ALTERNATE SPELLING FOR TERMS IN DATABASES

\begin{tabular}{|l|l|}
\hline American Spelling & \multicolumn{1}{|c|}{ British Spelling } \\
\hline Anesthesia & Anaesthesia \\
\hline Aging & Ageing \\
\hline Analyze & Analyse \\
\hline Center & Centre \\
\hline Favor & Favour \\
\hline Fetal & Foetal \\
\hline Hemorrhage & Haemorrhage \\
\hline Esophagus & Oesophagus \\
\hline Pediatrics & Paediatrics \\
\hline
\end{tabular}

